\title{
Correlation between Cohesive Energy Density, Fractional Free Volume, and Gas Transport Properties of Poly(ethylene-co-vinyl acetate) Materials
}

\author{
Piotr Kubica and Aleksandra Wolinska-Grabczyk \\ Centre of Polymer and Carbon Materials, Polish Academy of Sciences, Marie Curie-Sklodowskiej 34, 41-819 Zabrze, Poland \\ Correspondence should be addressed to Aleksandra Wolinska-Grabczyk; awolinska@cmpw-pan.edu.pl
}

Received 24 February 2015; Revised 25 April 2015; Accepted 19 May 2015

Academic Editor: Subrata Mondal

Copyright (C) 2015 P. Kubica and A. Wolinska-Grabczyk. This is an open access article distributed under the Creative Commons Attribution License, which permits unrestricted use, distribution, and reproduction in any medium, provided the original work is properly cited.

\begin{abstract}
The transport properties of the poly(ethylene-co-vinyl acetate) (EVA) materials to $\mathrm{He}, \mathrm{N}_{2}, \mathrm{O}_{2}$, and $\mathrm{CO}_{2}$ are correlated with two polymer molecular structure parameters, that is, cohesive energy density (CED) and fractional free volume (FFV), determined by the group contribution method. In our preceding paper, the attempt was made to approximate EVA permeability using a linear function of $1 / \mathrm{FFV}$ as predicted by the free volume theory. However, the deviations from this relationship appeared to be significant. In this paper, it is shown that permeation of gas molecules is controlled not only by free volume but also by the polymer cohesive energy. Moreover, the behavior of $\mathrm{CO}_{2}$ was found to differ significantly from that of other gases. In this instance, the correlation is much better when diffusivity instead of permeability is taken into account in a modified transport model.
\end{abstract}

\section{Introduction}

Gas transport properties of polymers are often correlated with their free volume defined as the excess volume which can be redistributed without energy change [1]. Average free volume can also be understood as a parameter representing the volume within the polymer, which is unoccupied by polymer chains [2]. Based on the free volume theory by Cohen and Turnbull [3], a relationship between coefficient of gas diffusion in polymer, $D$, and polymer fractional free volume, FFV, has been established:

$$
\ln D=a-\frac{b}{\mathrm{FFV}} .
$$

According to the solution-diffusion model of gas transport in nonporous materials, product of diffusion, $D$, and solubility, $S$, coefficients gives permeability, $P$, that is, gas flux through polymer film normalized by its thickness and pressure difference between both film sides. Since $S$ varies with free volume much less than $D$ does, it can be assumed that changes in permeability are similar to those in diffusivity $[2,4]$. If systems under investigation satisfy such assumption, that is exactly valid for light/inert gases such as helium or hydrogen, the following relationship between polymer permeability and fractional free volume should apply:

$$
\ln P=A-\frac{B}{\mathrm{FFV}},
$$

and the value of $B$ coefficient in (2) should be similar to that of $b$ from (1). In many works, the validity of these equations in describing and predicting gas transport properties of polymers has been investigated. There are studies showing that FFV correlates reasonably well with $D$ and $P$ of polymers [5-8]. When larger discrepancies between experimental results and theory were obtained, errors in FFV estimation or differences in FFV distribution across materials, which are not taken into account in (1) and (2), have been proposed as a possible explanation [9]. On the other hand, the behavior opposite to that predicted by the theory has been demonstrated. For example, it was found that insertion of some side-chains into polymer structure results in lower permeability despite the fact that FFV increases [10]. The authors explained this rather surprising effect by 
the fact that parallel to the FFV increase, side-chain insertion decreased the chain mobility as demonstrated by the enhanced glass transition temperature, $T_{\mathrm{g}}$, of those materials. Since the latter effect prevailed, diffusion and permeability coefficients were found to decrease. Apart from FFV and $T_{\mathrm{g}}$, cohesive energy density, CED, is another polymer property that can be correlated with gas transport. It was shown by Meares that activation energy of diffusion, $E_{\mathrm{d}}$, is proportional to CED of a polymer in which diffusion takes place [11]. This fact implies that diffusion coefficient and also permeability coefficient due to the relation $P=D S$ are both affected by CED.

Cohesive energy $\left(E_{\text {coh }}\right)$ of a substance is defined as an increase in internal energy per mole of a substance if all intermolecular forces are eliminated [12]. Thus, it is a measure of intermolecular forces strength. Dividing $E_{\text {coh }}$, expressed in $\mathrm{J} \mathrm{mol}^{-1}$, by molar volume $V$ of a substance $\left(\mathrm{cm}^{3} \mathrm{~mol}^{-1}\right)$, one obtains another property, cohesive energy density (CED) $\left(\mathrm{J} \mathrm{cm}^{-3}\right)[12]$. It was found that linear correlations of transport parameters with variables accounting simultaneously for both CED and FFV can give better results than the respective correlations with FFV or CED alone [13-16]. Alentiev and Yampolskii proposed the following relationship between $P$, $\mathrm{CED}$, and FFV [13]:

$$
\ln P=A^{\prime}-B^{\prime}\left(\frac{\mathrm{CED}}{\mathrm{FFV}}\right) .
$$

As shown by the authors, this relationship, though not derived theoretically, corresponds to the experimental observations that permeability of polymers is an increasing function of FFV and a decreasing function of CED. They also pointed out that the proposed equation does not contradict the physics of the diffusion process expressed by (i) the fundamental relationship of Cohen and Turnbull and (ii) Meares relationship predicting a linear dependence of $\ln D$ on $\mathrm{CED}$ when incorporated into the Arrhenius expression.

Recently, we presented the correlations between gas permeability and fractional free volume for a series of ethylenevinyl acetate (EVA) copolymers and their blends, varying in the vinyl acetate (VA) content [17]. In our work, we demonstrated that the experimental points obtained for most of the gases tested could not be satisfactorily approximated by a linear function of $1 / \mathrm{FFV}$. We proposed that the scatter of the points from linear behavior could be explained by the differences in FFV distribution in the EVA materials, which are not considered in (2). However, deeper insight into the observed phenomena has revealed that the deviations of the experimental data from theory may result from the differences in cohesive energy density among the investigated materials.

Therefore, in this paper an approach developed by Alentiev and Yampolskii [13] has been applied to interpret the results concerning gas transport in this group of polymer materials. While permeabilities of $\mathrm{He}, \mathrm{O}_{2}, \mathrm{~N}_{2}$, and $\mathrm{CO}_{2}$ in the EVA copolymers and blends and their physical characterizations have been reported in our previous work [17], diffusivity and solubility data obtained to clarify the issue are presented here for the first time.
Most of the EVA materials studied are semicrystalline materials, though the highest crystallinity degree did not exceed the value of $12 \%$ [17]. Crystallites are generally assumed to be nonpenetrable for small molecules and to constitute a hindrance to their motion through the system [4]. Michaels and Bixler [18] showed that for semicrystalline polyethylene gas solubility coefficient is directly proportional to the amorphous volume fraction of polymer, $X$ :

$$
S=S_{\mathrm{am}} X,
$$

where $S_{\mathrm{am}}$ is the solubility coefficient in completely amorphous material. To account for reduction of diffusion constant in semicrystalline polyethylene below the value expected for the totally amorphous polymer, Michaels and Parker [19] proposed two impedance factors, according to the expression

$$
D=\frac{D_{\mathrm{am}}}{\tau \beta},
$$

where $D_{\mathrm{am}}$ is the diffusion coefficient in completely amorphous material, $\tau$ is a geometrical impedance (tortuosity) factor, and $\beta$ is an immobilization factor. The tortuosity factor is a measure of the lengthening of the diffusive pathway associated with the necessity of molecules to bypass the crystallites, while $\beta$ represents the reduction in amorphous chain segment mobility in the vicinity of crystallites. Lasoski Jr. and Cobbs Jr. [20] studied permeability of polyethylene, poly(ethylene terephthalate), and nylon 610 to water vapor and found that $P$ increased as the polymer amorphous fraction $X$ was increased, following the relation

$$
P=P_{\mathrm{am}} X^{2} .
$$

Thus, assuming the validity of (4), one can obtain that

$$
D=D_{\text {am }} X .
$$

Equations (4), (6), and (7) are generally accepted as a first approximation of transport parameters in semicrystalline polymers [4], if any data concerning impedance factors are available. In this work, (6) and (7) have been applied to correlate permeability and diffusivity constants, and the validity of using them seems to be legitimate due to a very low volume fraction of crystallites in the EVA materials studied.

The application of the free volume-based model taking into account CED of the EVA materials along with new experimental findings has provided the basis of discussion of the differences in permeation behavior observed for different gas permeants.

\section{Experimental}

2.1. Materials and Membranes Formation. All membranes used for gas transport measurements were prepared from commercial EVA copolymers or from their mixture according to the procedure presented in our previous work [17]. The following EVA copolymers, EVA25, EVA31.5, EVA40, EVA46, EVA70, and the miscible blends of various compositions prepared from EVA31.5 and EVA70 [17] were studied. 
2.2. Measurements. Measurements of $\mathrm{CO}_{2}$ and $\mathrm{N}_{2}$ transport in the EVA materials were performed at $22^{\circ} \mathrm{C}$ using constant volume system. The procedure employed during measurements, adapted from literature [21], was as follows: after putting membrane into permeation cell, both upstream and downstream sides were evacuated (to pressure lower than $1 \mathrm{mmHg}$ ) and membrane was allowed to degas overnight. Directly before measurement, leak rate (i.e., pressure increase caused by the leakage of air into nonperfectly sealed system) was monitored. After that, feed gas was allowed to contact the membrane at pressure $(6 \pm 0.1)$ bar and pressure increase, in the range $0-10 \mathrm{mmHg}$, in the downstream side was recorded using $820 \mathrm{G}$ Series absolute pressure sensor. The process was conducted to achieve steady-state flux. Diffusion coefficients were calculated using the following formula:

$$
D=\frac{l^{2}}{6 \theta}
$$

where $l$ is the membrane thickness and $\theta$ is the time lag, that is, time obtained by extrapolation of the steady-state part of a pressure-time curve to $p=0$ ordinate. Permeability coefficients, $P$, were determined using the following formula:

$$
P=\frac{V_{\mathrm{d}} l}{p_{2} A T \mathrm{R}}\left[\left(\frac{\mathrm{d} p_{1}}{\mathrm{~d} t}\right)_{\mathrm{ss}}-\left(\frac{\mathrm{d} p_{1}}{\mathrm{~d} t}\right)_{\text {leak }}\right]
$$

where $V_{\mathrm{d}}$ is the downstream volume $\left(\mathrm{cm}^{3}\right), p_{2}$ and $p_{1}$ are feed and permeate pressure $(\mathrm{cmHg})$, respectively, $A$ is the membrane area $\left(\mathrm{cm}^{2}\right), \mathrm{R}$ is the gas constant $(\mathrm{cmHg}$ $\mathrm{cm}^{3} \mathrm{~cm}^{-3}$ (STP) $\mathrm{K}^{-1}$ ), $T$ is absolute temperature (K), at which measurements were carried out, and $t$ is time (s). The index "ss" means that the value of pressure increase with respect to time $\left(\mathrm{cmHg} \mathrm{s}^{-1}\right)$ was taken at steady-state conditions (to calculate this derivative, a part of a curve from 10 to 20 time lags was used), and the index "leak" refers to the rate of pressure increase during leak tests. In each experiment, leak rate was lower than two percent of the steady-state flux.

Additionally, measurements of the helium permeation rates were repeated, which allowed for more accurate data to be obtained since the previous paper [17], and these data are used here.

Measured quantities were recalculated for fully amorphous material using the data concerning volume fraction of the amorphous phase, taken from the previous work [17], and the formulae given by (6) and (7). The degree of crystallinity $(1-X)$ estimated for those materials ranged from 0.12 to 0 for EVA copolymers and from 0.09 to 0.02 for EVA blends.

From permeability and diffusivity data, gas solubility in the amorphous phase was obtained using the relationship that results from the solution-diffusion model:

$$
S_{\mathrm{am}}=\frac{P_{\mathrm{am}}}{D_{\mathrm{am}}} .
$$

2.3. Cohesive Energy Density Calculation. Cohesive energy of the investigated copolymers was calculated using the group contribution method. Contributions of appropriate chemical groups to cohesive energy (at $298 \mathrm{~K}$ ) were taken from the data

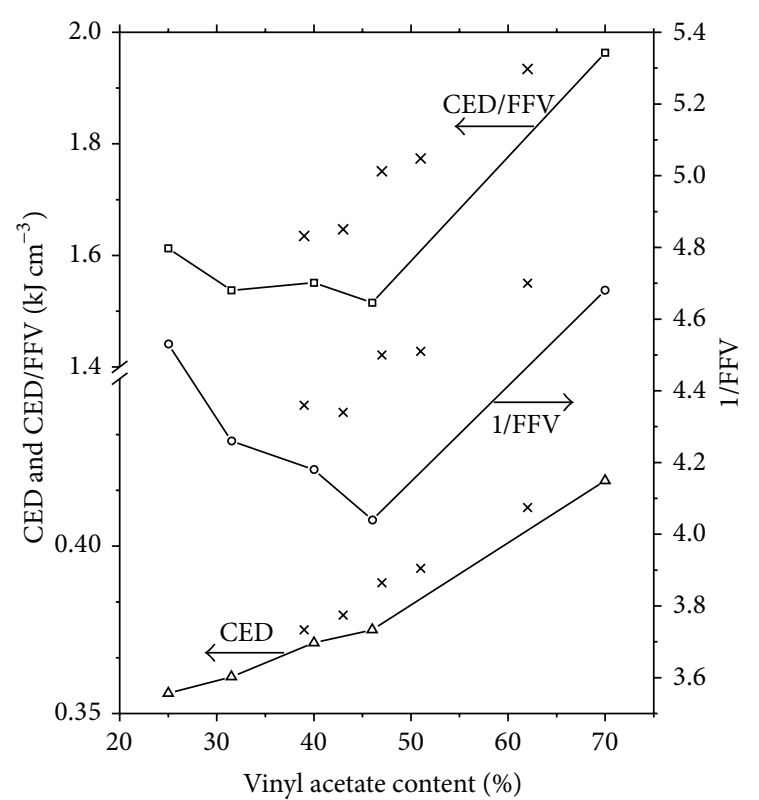

FIGURE 1: Dependence of CED and CED/FFV on VA content for pure EVA copolymers (empty symbols) and for copolymer blends (stars). Dependence of 1/FFV was plotted based on the data from the previous work [17] with permission from A. Wolinska-Grabczyk, P. Kubica, A. Jankowski, and J. Membrane Sci., 443, 227-236, Elsevier, copyright 2013.

collected by Fedors and presented by van Krevelen [12, 22]. The respective values in $\mathrm{J} \mathrm{mol}^{-1}$ were used: 4940 for $-\mathrm{CH}_{2}$ group, 17370 for -CO-, 3350 for -O-, 4710 for $-\mathrm{CH}_{3}$, and 3430 for $-\mathrm{CH}<$. From these data, contributions of ethylene, $E_{\mathrm{coh}}(\mathrm{Et})$, and vinyl acetate, $E_{\mathrm{coh}}(\mathrm{VA})$, units were calculated. Assuming that the repeat unit of EVAx contains $x$ vinyl acetate and $(1-x)$ ethylene units, cohesive energy of EVAx was calculated as

$$
E_{\mathrm{coh}}(\mathrm{EVA} x)=x E_{\mathrm{coh}}(\mathrm{VA})+(1-x) E_{\mathrm{coh}}(\mathrm{Et}) .
$$

To obtain CED, the above equation was divided by molar volume $V$ given by the following expression:

$$
V(\operatorname{EVA} x)=\frac{x M(\mathrm{VA})+(1-x) M(\mathrm{Et})}{d},
$$

where $M$ is the molar mass of a particular unit and $d$ is the density of a given copolymer, recalculated for its amorphous fraction, which was taken from the previous work [17]. The same procedure was used to calculate CED of the EVA blends, for which VA content was taken as $70 w_{\operatorname{EVA70}}+31.5(1-$ $w_{\text {EVA70 }}$ ), where $w_{\text {EVA70 }}$ is the weight fraction of EVA70 in its blend with EVA31.5. The obtained CED values, along with the FFV ones calculated previously [17], were used to calculate the $\mathrm{CED} / \mathrm{FFV}$ ratio for each EVA material.

\section{Results and Discussion}

Figure 1 presents dependence of CED, 1/FFV, and CED/FFV on vinyl acetate content for the pure EVA copolymers and for the blends of copolymers. 


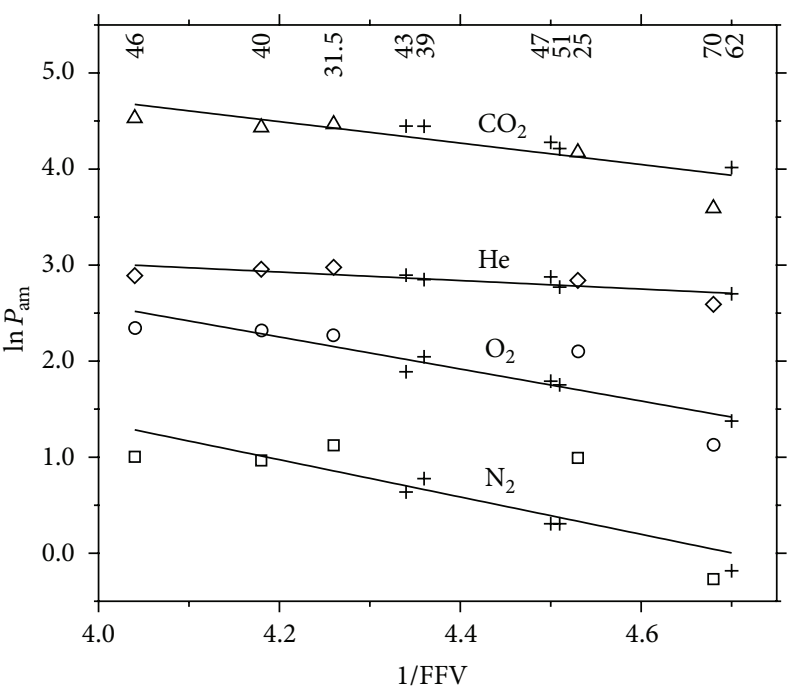

(a)

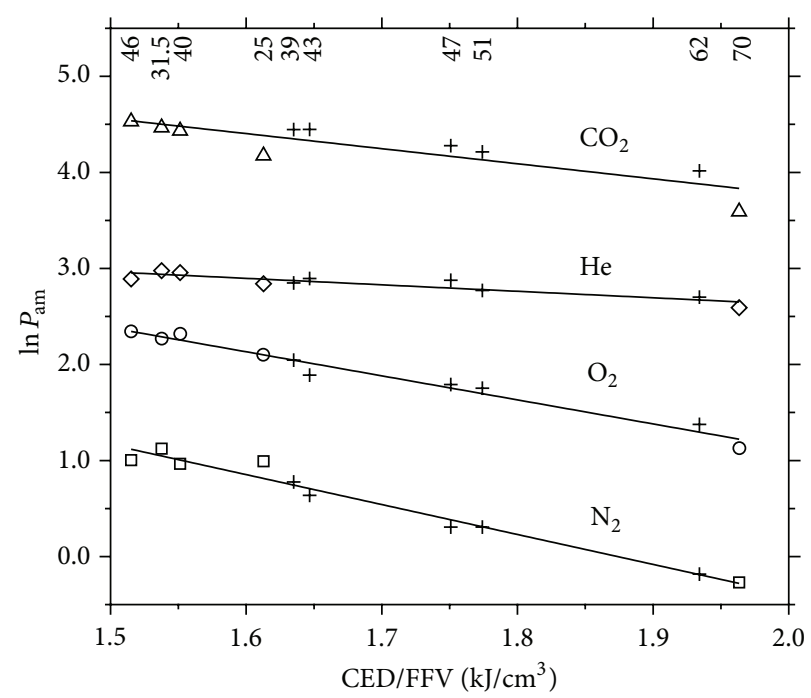

(b)

FIGURE 2: Correlation of permeability with (a) 1/FFV and (b) CED/FFV for the EVA based materials including both pure EVA copolymers (empty points) and their blends (+). Straight lines represent linear regressions of the experimental data. Numbers on the top axis represent vinyl acetate content. The plots for $\mathrm{CO}_{2}, \mathrm{O}_{2}$, and $\mathrm{N}_{2}$ shown in (a) are based on the data taken from our previous work [17] with permission from A. Wolinska-Grabczyk, P. Kubica, A. Jankowski, and J. Membrane Sci., 443, 227-236, Elsevier, copyright 2013.

It can be seen from this figure that CED increases monotonically with vinyl acetate content. This effect can be easily explained by the high cohesive energy of a vinyl acetate unit, due to a very high $E_{\text {coh }}$ of the carbonyl group as demonstrated by the values listed in the previous section. Another quantity presented in Figure 1, the reciprocal of fractional free volume (1/FFV), possesses a minimum value at $46 \%$ of vinyl acetate. Based on the cohesive energy density behavior, one might expect the monotonic decrease in the fractional free volume (thus monotonic increase in 1/FFV) without any extremum. However, some of the EVA materials contain crystalline parts, the fraction of which decreases from 0.12 for EVA25 to 0.02 for EVA 46 [17]. The increasing mobility of polymer chain segments caused by diminishing crystallinity between $25 \%$ and $46 \%$ of VA can be given as a reason for the increasing FFV values in this range. The curve representing dependence of CED/FFV on vinyl acetate content reflects the behavior of both CED dependence and 1/FFV dependence. Thus, in contrast to 1/FFV, CED/FFV ratio for copolymers decreases only slightly from 25 to $46 \%$ of VA. Considering the data shown in Figure 1, it can be also noticed that $\mathrm{CED} / \mathrm{FFV}$ value for EVA70 is nearly $22 \%$ higher than that for EVA25, whereas there is only a $3 \%$ difference in $1 / \mathrm{FFV}$ values between both materials. It means that these materials differ from one another much more than it appears from their 1/FFV quantities alone. From Figure 1, it can also be seen that for the EVA blends all three quantities follow nearly linear dependence on VA content.

Figure 2 presents the natural logarithm of EVA amorphous phase permeability as a function of CED/FFV values calculated in this work and as a function of $1 / \mathrm{FFV}$ values for comparison. Each CED/FFV or 1/FFV value refers to an individual EVA material as indicated on the top axis. For
TABLE 1: Parameters of (2) and (3) obtained from linear regression of the experimental data.

\begin{tabular}{lccccccc}
\hline \multirow{2}{*}{ Gas } & \multirow{2}{*}{$d_{\mathrm{k}}{ }^{(\mathrm{a})}[\AA]$} & \multicolumn{3}{c}{ Parameters of (2) } & \multicolumn{3}{c}{ Parameters of (3) } \\
& & $A$ & $B$ & $R^{2}$ & $A^{\prime}$ & $B^{\prime}$ & $R^{2}$ \\
\hline $\mathrm{He}$ & 2.6 & 4.8 & 0.4 & 0.60 & 4.0 & 0.7 & 0.81 \\
$\mathrm{CO}_{2}$ & 3.3 & 9.2 & 1.1 & 0.66 & 6.9 & 1.6 & 0.74 \\
$\mathrm{O}_{2}$ & 3.46 & 9.3 & 1.7 & 0.74 & 6.1 & 2.5 & 0.97 \\
$\mathrm{~N}_{2}$ & 3.64 & 9.1 & 1.9 & 0.63 & 5.8 & 3.1 & 0.97 \\
\hline
\end{tabular}

${ }^{(a)}$ Kinetic diameter of a gas molecule.

both models, one linear fit to the all experimental points was performed for a given gas. From the fit parameters, coefficients $A$ and $B$ present in (2) $(\ln P=f(1 / \mathrm{FFV}))$ and coefficients $A^{\prime}$ and $B^{\prime}$ present in (3) $(\ln P=f(\mathrm{CED} / \mathrm{FFV}))$ were calculated and collected in Table 1 . The values in Table 1 show that both $B$ and $B^{\prime}$ increase with a kinetic diameter of a gas molecule. This observation is consistent with the theory, which relates parameter $B\left(B^{\prime}\right)$ to the size of a penetrant molecule [3] and with general trends reported for other systems $[2,13]$. The explanation for such an effect is that larger gas molecules are more sensitive to changes in polymer structure (represented by changes in CED and FFV) than the smaller ones. When $B^{\prime}$ and $B$ parameters are plotted as a function of the gas molecule volume calculated using kinetic diameter values (Figure 3), linear correlations can be observed. Comparing the parameters resulting from linear fits to both data sets, one can notice a stronger dependence of $B^{\prime}$ on $d_{\mathrm{k}}{ }^{3}$, expressed by a greater slope of the regression line $(0.08$ versus 0.05$)$. This indicates that CED of the polymers plays an important role in the transport of small molecules. To further discuss the effect of CED, analyses of 


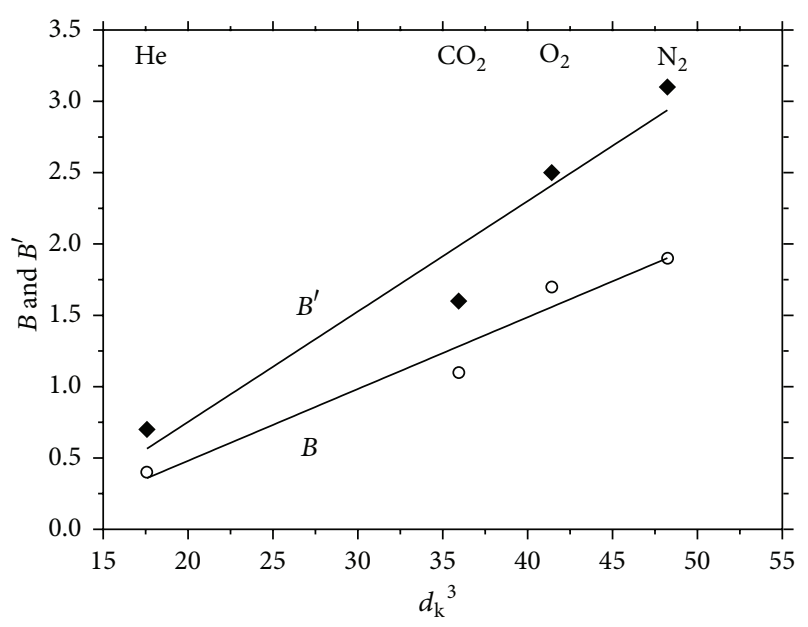

FIgURE 3: Dependence of $B$ and $B^{\prime}$ parameters on gas molecule volume $\left(d_{\mathrm{k}}{ }^{3}\right)$.

the coefficients of determination, $R^{2}$, resulting from linear regressions based on (2) and (3), have been carried out.

From the coefficients of determination, $R^{2}$, listed in Table 1, it can be noted that permeability of the EVA materials to $\mathrm{N}_{2}$ and $\mathrm{O}_{2}$ can be much better described by a linear function of $\mathrm{CED} / \mathrm{FFV}$ than by that of $1 / \mathrm{FFV}$. It shows that CED is a significant factor influencing permeation of these molecules. The effect of CED can also be seen when comparing the permeability of EVA25 and that of a blend containing $51 \%$ of VA. As demonstrated by the data in Figure 2, both materials possess nearly the same FFV, but due to its higher CED the blend is significantly less permeable to $\mathrm{N}_{2}$ and $\mathrm{O}_{2}$ than EVA25. Both materials reveal, however, similar permeability to helium, indicating that permeation of this gas is less affected by CED than permeation of $\mathrm{N}_{2}$ and $\mathrm{O}_{2}$. On the other hand, (3) fits the data for helium better than (2), though greater scatter of the experimental points is observed. The fact that transport of helium is the one the least dependent on CED quantity is also demonstrated by the lowest $B^{\prime}$ parameter value. The possible explanation of the He permeation behavior may be proposed based on considerations presented by Kumins and Kwei [23]. According to the transition state theory of diffusion, activation energy of diffusion can be expressed by the following equation:

$$
\Delta E_{\mathrm{d}}=\Delta E+p_{\mathrm{i}} \Delta V+\mathrm{R} T,
$$

where $\Delta E$ is the internal energy of activation, $p_{\mathrm{i}} \Delta V$ is the work required to provide $\Delta V$ volume increase between polymer chains, which is necessary for the diffusion of penetrant to take place, $R$ is the ideal gas constant, and $T$ is the absolute temperature. The work expressed by the second term depends on the extent of the volume increase $\Delta V$ and on the internal pressure $p_{\mathrm{i}}$ of the polymer. This dependence results from the fact that the work of creation of a free volume is performed against molecular interactions between polymer chains, which are reflected in internal pressure or CED (these quantities are closely related [12]). Following Kumins and Kwei's reasoning, small gases require a relatively small increase in volume; thus, the term $p_{\mathrm{i}} \Delta V$ is relatively small and the main contribution to $\Delta E_{\mathrm{d}}$ is the $\Delta E+\mathrm{RT}$ term, which is independent of CED. Therefore, changes in $E_{\mathrm{d}}$, resulting from the changes in polymer CED, are relatively small for small gases and this may explain why helium permeation investigated in this study is less dependent on CED. In the case of larger oxygen and nitrogen molecules, the necessary volume increase is larger, making the work term in (13) a significant contribution to the energy of activation. Thus, permeation of both gases is significantly affected by the changes in CED as demonstrated by the data in Figure 2, as well as by the $B^{\prime}$ and $R^{2}$ values presented in Table 1 . Similar results to those obtained in this work have been presented in the literature with respect to various glassy polymers $[15,16]$. In the work by Thran et al. [15], correlations of gas diffusion coefficient values with FFV determined by the group contribution method for a wide range of glassy polymers were analyzed. The correlations obtained allowed authors to claim that FFV is not the optimum polymer property to predict diffusion coefficient in polymers. They pointed out that incorporation of a third, depending on CED, term to (2) gave an equation representing experimental data better than a simple free volume model did. Based on the correlations between deviations of experimental points from regression lines for different gases, the authors proposed also that the mechanism of diffusion for small helium and hydrogen differs from that for the larger molecules and related this difference to the diffusant-dependent free volume. Similar conclusions can be found in the work by Nagel et al. concerning studies on the relationship between free volume determined by positron annihilation lifetime spectroscopy (PALS) and gas diffusion in a series of polyimides [16]. In both works, any pronounced differences were observed in the diffusion behavior for larger molecules, that is, $\mathrm{N}_{2}, \mathrm{O}_{2}, \mathrm{CO}_{2}$, and $\mathrm{CH}_{4}$. However, Thran et al. noticed a little different behavior of $\mathrm{CO}_{2}$ with regard to $\mathrm{N}_{2}, \mathrm{O}_{2}$, and $\mathrm{CH}_{4}$ [15]. He explained it as resulting either from the slightly different diffusion mechanism caused by the less spherical shape and more polar nature of the $\mathrm{CO}_{2}$ molecule compared to other gases or from the scatter of the $\mathrm{CO}_{2}$ diffusion coefficient values due to tendency of $\mathrm{CO}_{2}$ to plasticize polymers. On the other hand, Jia and Xu presented a linear relationship between $\log P$ and the ratio of specific free volume and cohesive energy obtained for 60 polymers of various structures and for all the gases studied, including $\mathrm{CO}_{2}[14]$. However, they admitted that the scattering of the data was considerable and ascribed it to the variety of the experimental permeability data sources.

The results of our studies, as demonstrated in Figure 2 or in Table 1, show that permeation behavior of $\mathrm{CO}_{2}$ is distinct from that observed for the other gases. From the analysis of the determination coefficient values of the linear fit to the $\mathrm{CO}_{2}$ permeability, given in Table 1 , it can be found that both (2) and (3) approximate $\mathrm{CO}_{2}$ permeability data with a similar goodness. The model involving CED fits the data only slightly better and the approximation is less satisfying than for oxygen and nitrogen. Taking into account chemical structure of both $\mathrm{CO}_{2}$ and EVA polymer and the results obtained, the necessity to perform more profound studies 


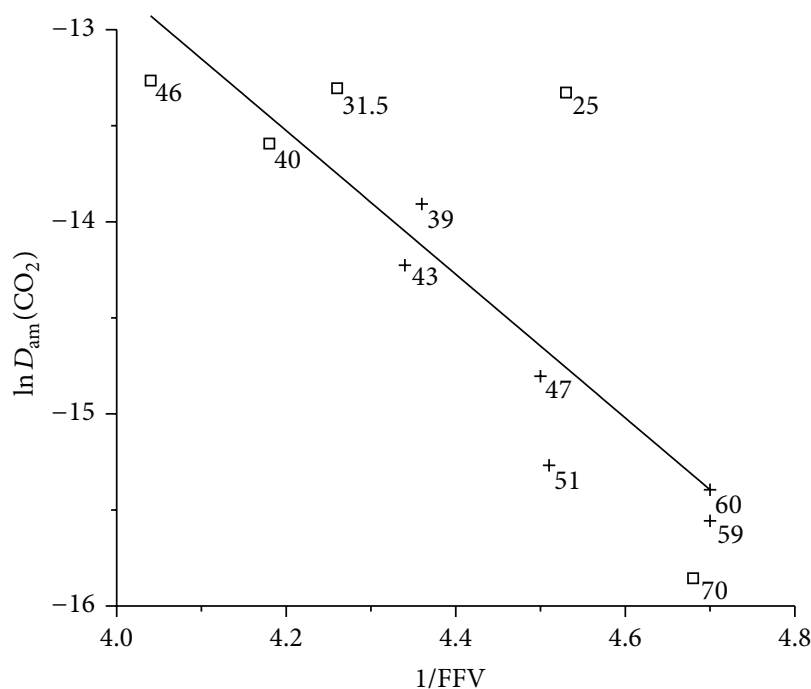

(a)

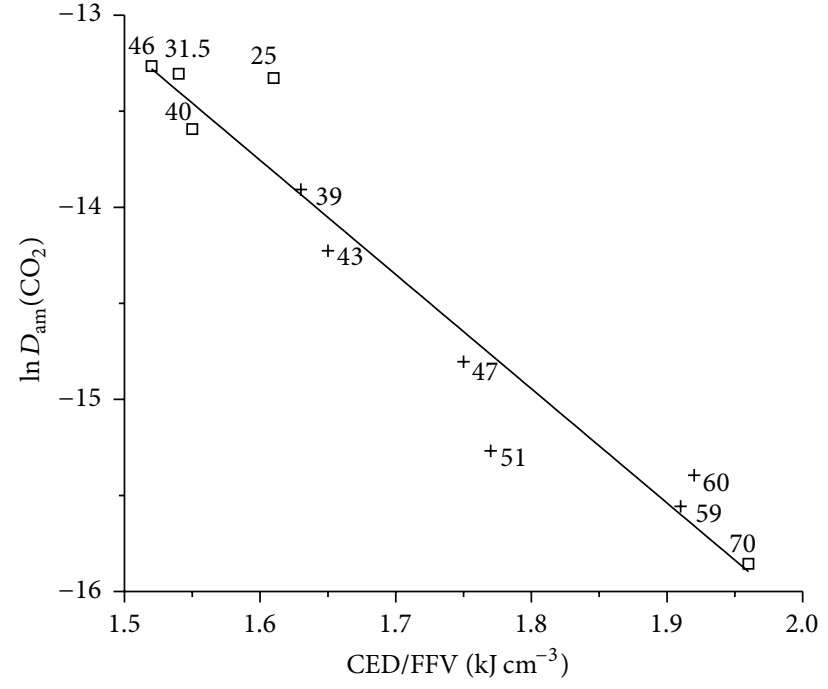

(b)

FIGURE 4: Correlation of the $\mathrm{CO}_{2}$ diffusion coefficients with (a) 1/FFV and (b) CED/FFV for both pure EVA copolymers ( $\square$ ) and their blends $(+)$. Numbers next to the points indicate vinyl acetate content in a given material. Straight lines represent linear regression of the experimental data.

concerning $\mathrm{CO}_{2}$ permeation appeared. From the literature, it is known that the solubility of $\mathrm{CO}_{2}$ in the EVA copolymers increases with the increasing vinyl acetate content [24]. Such an effect was ascribed to the presence of specific interactions, most probably of Lewis acid-base character, between $\mathrm{CO}_{2}$ molecules and electron donating acetate groups [25]. Additionally, electrostatic forces were also suggested as those which may contribute to these interactions, since $\mathrm{CO}_{2}$ molecules possess large quadrupole moment [25]. Because $\mathrm{CO}_{2}$ solubility changes with a vinyl acetate content, it is probable that the assumption, on which (2) and (3) are based, that is, small gas solubility variations, does not hold for $\mathrm{EVA} / \mathrm{CO}_{2}$ systems. Thus, in the case of $\mathrm{CO}_{2}$, the dependence of diffusion coefficient, $D$, rather than that of permeability should be more relevant. For simple free volume model, it means using (1) instead of (2), and for the model proposed by Alentiev and Yampolskii [13], the following relationship is to be considered:

$$
\ln D=a^{\prime}-b^{\prime}\left(\frac{\mathrm{CED}}{\mathrm{FFV}}\right) .
$$

To further discuss the applicability of both models to describe transport of $\mathrm{CO}_{2}$, its diffusion coefficients in the investigated materials were measured. Additionally, diffusion coefficients of nitrogen, an inert gas for which solubility is expected to be more or less constant, were also determined. In Figure 4, the $\mathrm{CO}_{2}$ diffusion coefficient values, recalculated for the amorphous phase fraction according to (7), are shown as a function of $1 / \mathrm{FFV}$ and CED/FFV. In this figure, straight lines representing linear fit to the experimental data are also shown. From the fitting parameters, coefficients of (1) and (14) were calculated and listed in Table 2. Based on the determination coefficients values, it may be found that the model which takes into account the materials CED describes $\mathrm{CO}_{2}$ diffusivity data much better than a simple free volume
TABLE 2: Parameters of (1) and (14) obtained from linear regression of the experimental data presented in Figures 4(a) and 4(b), respectively.

\begin{tabular}{lccccc}
\hline & Parameters of (1) & \multicolumn{4}{c}{ Parameters of (14) } \\
$a$ & $b$ & $R^{2}$ & $a^{\prime}$ & $b^{\prime}$ & $R^{2}$ \\
\hline 2.2 & 3.7 & 0.65 & -4.2 & 5.9 & 0.93 \\
\hline
\end{tabular}

model. Thus, considering the permeability results for $\mathrm{N}_{2}$ and $\mathrm{O}_{2}$, and the results for $\mathrm{CO}_{2}$ diffusivity, it may be concluded that transport of these gases is significantly affected by both FFV and CED. Similarly, permeation of helium, despite being less dependent on both quantities, is better approximated by the model involving CED.

Additionally, the results obtained prove that $\mathrm{CO}_{2}$ permeability variation among investigated materials is significantly affected by variations in its solubility. The comparison of $B^{\prime}(\ln P$ versus $\mathrm{CED} / \mathrm{FFV})$ and $b^{\prime}(\ln D$ versus CED/FFV) parameters for $\mathrm{CO}_{2}$ transport, which are 1.6 and 5.9, respectively, reveals that diffusivity changes with CED/FFV much more than permeability does. If solubility does not vary significantly, then the slopes of the regression lines, $B^{\prime}$ and $b^{\prime}$, should be similar because more or less constant $S$ provides more or less constant shift to each experimental point, when diffusivity is replaced by permeability. This is confirmed by the data obtained for nitrogen, which is an inert gas, interacting very weakly with macromolecules, and the solubility of which is not expected to vary considerably among the EVA materials. Figure 5 presents the correlation of $\mathrm{N}_{2}$ diffusion coefficient in the EVA materials with $\mathrm{CED} / \mathrm{FFV}$ ratio, whereas the results of linear regression of the experimental data are given in the caption of this figure. As can be seen, the parameters $B^{\prime}(\ln P$ versus CED/FFV) 


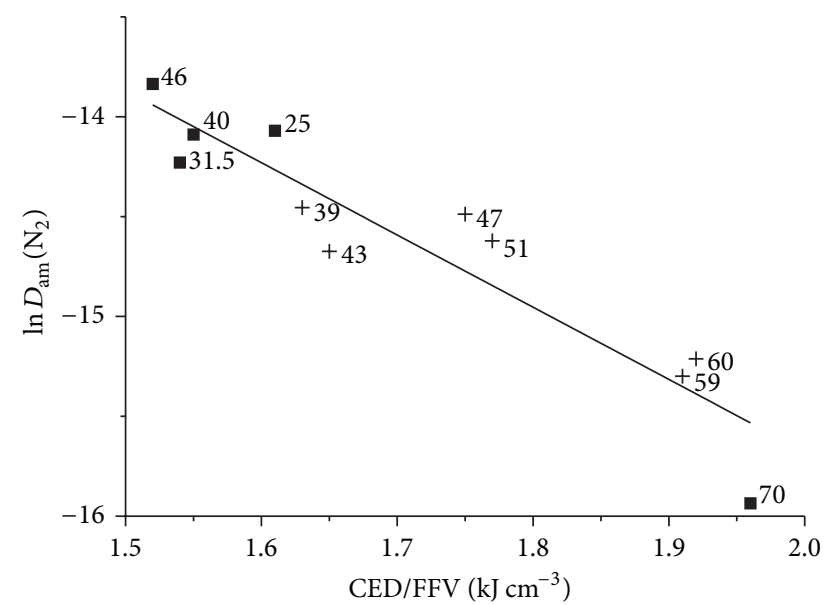

Figure 5: Correlation of $\mathrm{N}_{2}$ diffusion coefficient for (匹) EVA copolymers and for (+) blends. The straight line represents linear fit to the experimental data. The obtained parameters of (14) are $a^{\prime}=-8.4, b^{\prime}=3.6$, and $R^{2}=0.86$.

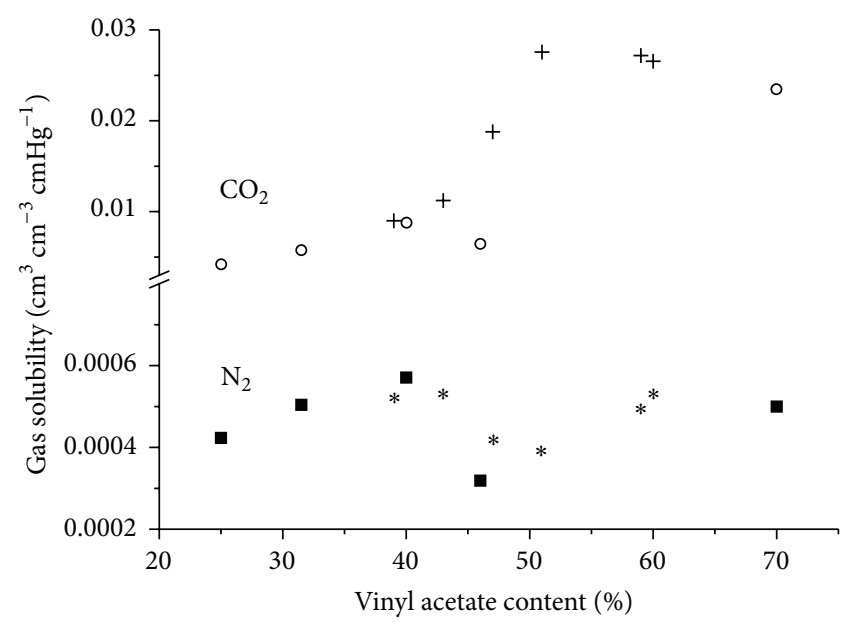

FIGURE 6: Amorphous phase gas solubility variations with VA content in EVA copolymers $(\circ, \boldsymbol{\square})$ and EVA copolymer blends $(+, *)$. Note that different scales below and above break on the vertical axis were used.

and $b^{\prime}\left(\ln D\right.$ versus CED/FFV) for $\mathrm{N}_{2}$ transport, which are 3.1 and 3.6, respectively, differ from one another only by $16 \%$. This is much less than the almost fourfold difference between those parameters determined for $\mathrm{CO}_{2}$ transport.

To illustrate the solubility differences between $\mathrm{CO}_{2}$ and $\mathrm{N}_{2}$ in the investigated materials, the permeability and diffusivity data have been used to calculate the solubility coefficients values according to (10). The calculated values plotted as a function of the vinyl acetate content are shown in Figure 6. As can be seen from this figure, $\mathrm{CO}_{2}$ solubility varies as much as by a factor of 6 among the EVA materials, whereas the differences in nitrogen solubility are significantly smaller and do not exceed a factor of 1.7.

\section{Conclusions}

It was found that transport of small gases such as $\mathrm{N}_{2}, \mathrm{O}_{2}$, $\mathrm{He}$, and $\mathrm{CO}_{2}$ through EVA copolymers can be much better described by a model taking into account cohesive energy density of the materials than by a simple free volume model. Moreover, the results obtained show that the variations in the amount of the specific functional groups present in the polymers limit the applicability of (2) and (3) to describe transport of gases such as $\mathrm{CO}_{2}$, able to interact with those groups. For those systems, the respective correlations should be used in their basic form, involving diffusion coefficient instead of the permeability one. Based on the correlations discussed, it can also be concluded that the methods used to calculate FFV and CED do not introduce large errors to these quantities allowing for estimation of their dependence on vinyl acetate content, for both EVA copolymers and their blends, with rather good accuracy.

\section{Conflict of Interests}

The authors declare that there is no conflict of interests regarding the publication of this paper.

\section{Acknowledgments}

This work was partially financed by the Grant no. N N209 086 138 given by the Ministry of Science and Higher Education of Poland. P. Kubica acknowledges the support from the DoktoRIS project-scholarship program for the innovation of Silesia region supported by the European Community from the European Social Fund.

\section{References}

[1] D. Turnbull and M. H. Cohen, "Free-volume model of the amorphous phase: glass transition," The Journal of Chemical Physics, vol. 34, no. 1, p. 120, 1961.

[2] S. Matteucci, Y. Yampolskii, B. D. Freeman, and I. Pinnau, "Transport of gases and vapors in glassy and rubbery polymers," in Materials Science of Membranes for Gas and Vapor Separation, Y. Yampolskii, I. Pinnau, and B. D. Freeman, Eds., chapter 1, John Wiley \& Sons, Chichester, UK, 2006.

[3] M. H. Cohen and D. Turnbull, "Molecular transport in liquids and glasses," The Journal of Chemical Physics, vol. 31, no. 5, pp. 1164-1169, 1959.

[4] D. W. van Krevelen and K. te Nijenhuis, "Properties determining mass transfer in polymeric systems," in Properties of Polymers. Their Correlation with Chemical Structure; Their Numerical Estimation and Prediction from Additive Group Contributions, chapter 18, Elsevier, Amsterdam, The Netherlands, 4th edition, 2009.

[5] M. R. Pixton and D. R. Paul, "Relationships between structure and transport properties for polymers with aromatic backbones," in Polymeric Gas Separation Membranes, D. R. Paul and Y. P. Yampolskii, Eds., chapter 3, CRC Press, Boca Raton, Fla, USA, 1994

[6] W. Li, S. Zhang, G. Chen, and Q. Zhang, "Synthesis and properties of novel fluorinated poly(phenylene-co-imide)s," 
Macromolecular Chemistry and Physics, vol. 208, no. 3, pp. 307315, 2007.

[7] H. Lin and B. D. Freeman, "Gas permeation and diffusion in cross-linked poly(ethylene glycol diacrylate)," Macromolecules, vol. 39, no. 10, pp. 3568-3580, 2006.

[8] A. C. Puleo, N. Muruganandam, and D. R. Paul, "Gas sorption and transport in substituted polystyrenes," Journal of Polymer Science. Part B: Polymer Physics, vol. 27, no. 11, pp. 2385-2406, 1989.

[9] Y. Yampolskii and V. Shantarovich, "Positron annihilation lifetime spectroscopy and other methods for free volume evaluation in polymers," in Materials Science of Membranes for Gas and Vapor Separation, Y. Yampolskii, I. Pinnau, and B. D. Freeman, Eds., chapter 6, John Wiley \& Sons, Chichester, UK, 2006.

[10] V. A. Kusuma, S. Matteucci, B. D. Freeman, M. K. Danquah, and D. S. Kalika, "Influence of phenoxy-terminated short-chain pendant groups on gas transport properties of cross-linked poly(ethylene oxide) copolymers," Journal of Membrane Science, vol. 341, no. 1-2, pp. 84-95, 2009.

[11] P. Meares, "The diffusion of gases through polyvinyl acetate," Journal of the American Chemical Society, vol. 76, no. 13, pp. 3415-3422, 1954.

[12] D. W. Van Krevelen and K. Te Nijenhuis, "Chapter 7: cohesive properties and solubility," in Properties of Polymers: Their Correlation with Chemical Structure: Their Numerical Estimation and Prediction from Additive Group Contributions, Elsevier, Amsterdam, The Netherlands, 4th edition, 2009.

[13] A. Y. Alentiev and Y. P. Yampolskii, "Meares equation and the role of cohesion energy density in diffusion in polymers," Journal of Membrane Science, vol. 206, no. 1-2, pp. 291-306, 2002.

[14] L. Jia and J. Xu, "A simple method for prediction of gas permeability of polymers from their molecular structure," Polymer Journal, vol. 23, no. 5, pp. 417-425, 1991.

[15] A. Thran, G. Kroll, and F. Faupel, "Correlation between fractional free volume and diffusivity of gas molecules in glassy polymers," Journal of Polymer Science Part B: Polymer Physics, vol. 37, no. 23, pp. 3344-3358, 1999.

[16] C. Nagel, K. Günther-Schade, D. Fritsch, T. Strunskus, and F. Faupel, "Free volume and transport properties in highly selective polymer membranes," Macromolecules, vol. 35, no. 6, pp. 2071-2077, 2002.

[17] A. Wolinska-Grabczyk, P. Kubica, and A. Jankowski, "Effect of the acetate group content on gas permeation through membranes based on poly(ethylene-co-vinyl acetate) and its blends," Journal of Membrane Science, vol. 443, pp. 227-236, 2013.

[18] A. S. Michaels and H. J. Bixler, "Solubility of gases in polyethylene," Journal of Polymer Science, vol. 50, no. 154, pp. 393-412, 1961.

[19] A. S. Michaels and R. B. Parker Jr., "Sorption and flow of gases in polyethylene," Journal of Polymer Science, vol. 41, no. 138, pp. 53-71, 1959.

[20] S. W. Lasoski Jr. and W. H. Cobbs Jr., "Moisture permeability of polymers. I. Role of crystallinity and orientation," Journal of Polymer Science, vol. 36, no. 130, pp. 21-33, 1959.

[21] B. D. Freeman and H. Lin, "Permeation and diffusion," in Springer Handbook of Materials Measurement Methods, $\mathrm{H}$. Czichos, T. Saito, and L. Smith, Eds., pp. 371-387, Springer, 2006.

[22] R. F. Fedors, "A method for estimating both the solubility parameters and molar volumes of liquids," Polymer Engineering \& Science, vol. 14, no. 2, pp. 147-154, 1974.
[23] C. A. Kumins and T. K. Kwei, "Free volume and other theories," in Diffusion in Polymers, J. Crank and G. S. Park, Eds., chapter 4, Academic Press, London, UK, 1968.

[24] S. Marais, J. M. Saiter, C. Devallencourt, Q. T. Nguyen, and M. Métayer, "Study of transport of small molecules through ethylene-co-vinyl acetate copolymers films. Part B: $\mathrm{CO}_{2}$ and $\mathrm{O}_{2}$ gases," Polymer Testing, vol. 21, no. 4, pp. 425-431, 2002.

[25] S. G. Kazarian, M. F. Vincent, F. V. Bright, C. L. Liotta, and C. A. Eckert, "Specific intermolecular interaction of carbon dioxide with polymers," Journal of the American Chemical Society, vol. 118, no. 7, pp. 1729-1736, 1996. 

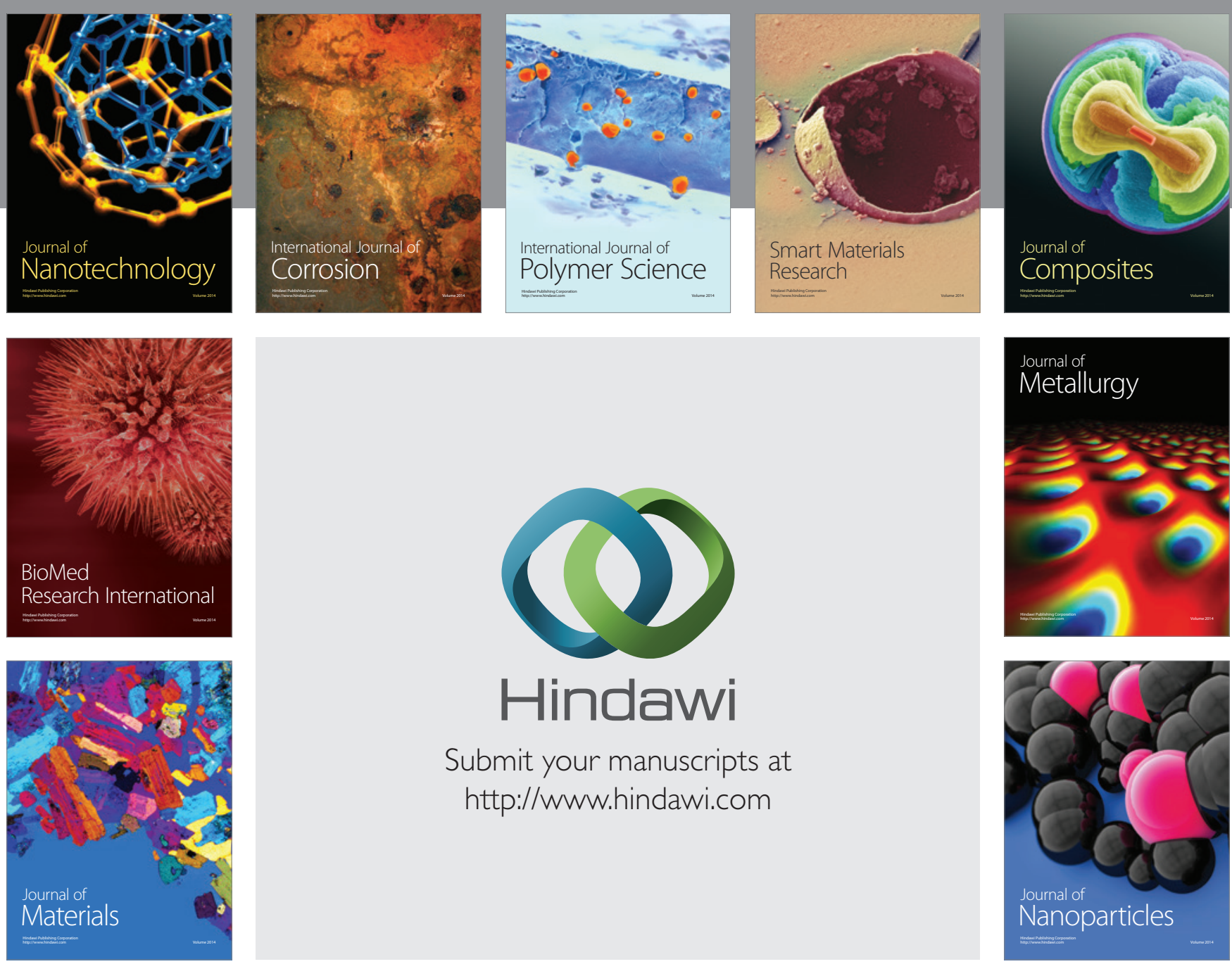

Submit your manuscripts at http://www.hindawi.com
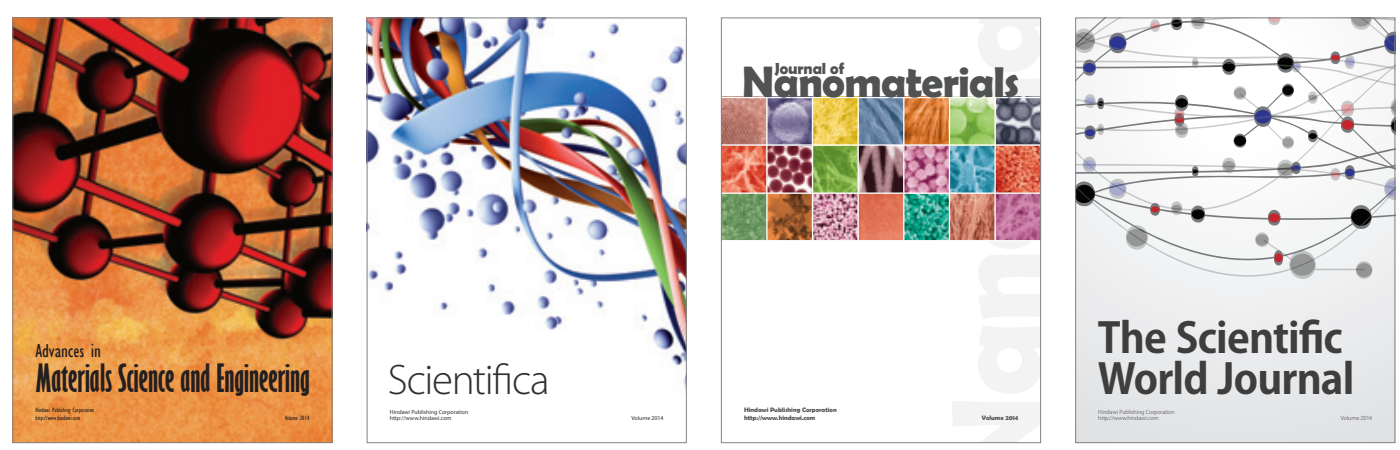

\section{The Scientific World Journal}
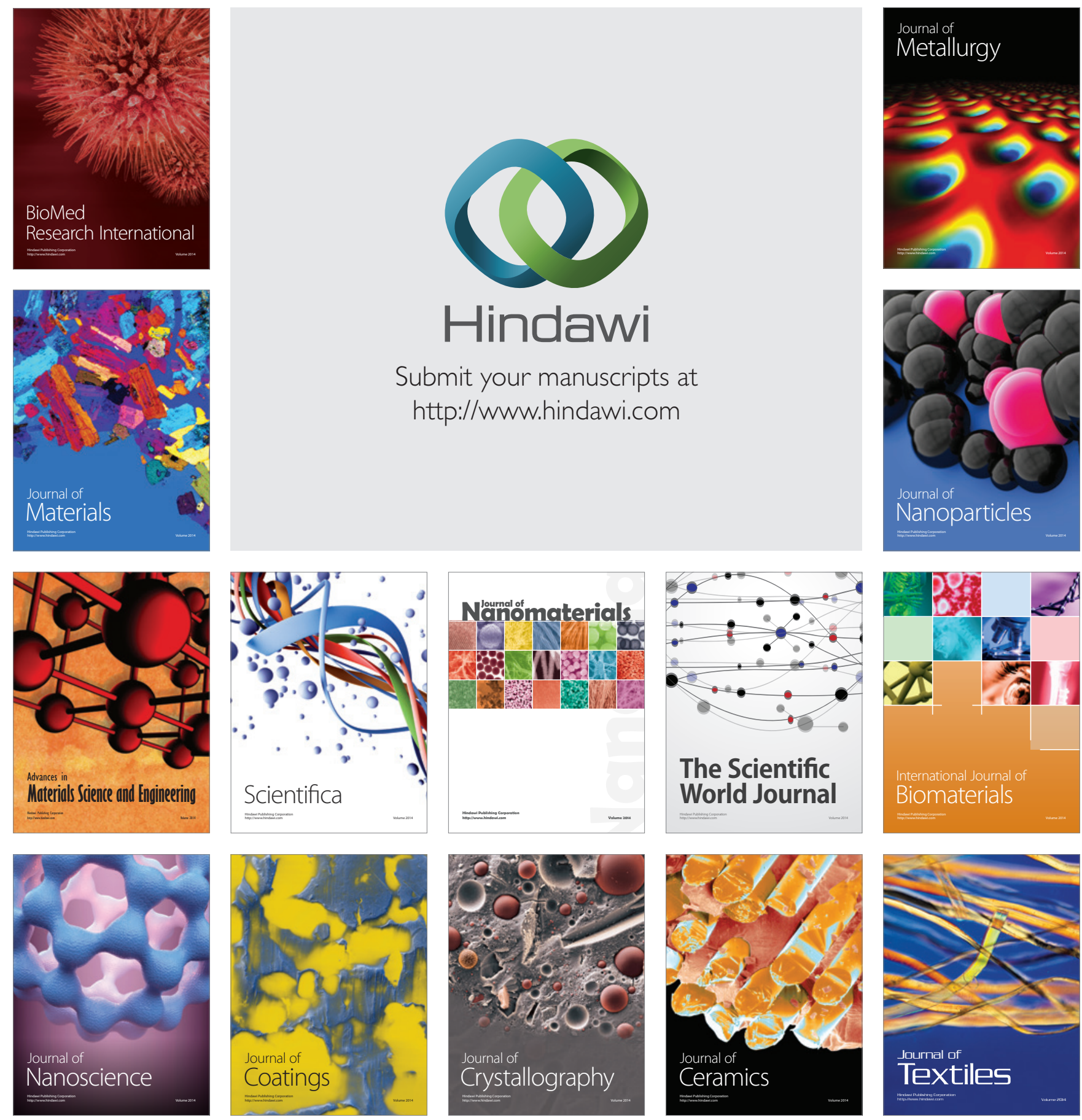